\title{
TENTATIVE ORDER OF DIFFICULTY OF THE TERMAN VOCABULARY WITH VERY YOUNG CHILDREN
}

\author{
MARGARET V. COBB
}

Institute of Educational Research, Teachers College, Columbia University

In the course of a study of group intelligence tests in Grade I, which involved Binet examining, it became evident that the words in the Vocabulary Test (Stanford Revision) show an order of difficulty for small children which is noticeably different from that indicated on the blank. Tabulation of the scores on the separate words gave the following information, which may prove useful to others who are examining kindergarten and Grade I children.

The examining was done by five persons, including myself, the others being Miss Helen Davis, Director of Measurements and Special Education in the public schools of Jackson, Michigan, through whose courtesy the records were made available, and three of the kindergarten teachers of Jackson whom she had trained for the work and who had shown special aptitude for it. The children were examined in the kindergarten during the first semester of the school year 1920-1921, and in Grade I during the second semester of the same year. With a few children the test was begun with word 6 in each list; the previous words were omitted but given credit, if there were no failures before word 11 ("Roar" and "haste" were, however, always given.). The test was stopped when the child missed five successive words in each list. Three or four of the scoring conventions observed in addition to the rules in "The Measurement of Intelligence" should probably be noted here, since a different rule might have altered the result:

Bonfire.-Full credit, any definition which gave a hint of distinction between a bonfire and other fire, as "A fire outdoors," "A big fire," "Go burn things up," etc. Half credit, definitions of fire alone, as "Furnace fire," "Fire," "In a stove," etc.

Haste.-Full credit for definition involving hurry or speed. Half credit for quotation from school song ("haste away," etc.) or definition as "go," "fly," etc.

Afloat.-Full cedit for definition involving floating on surface. Half credit for definition as moving along, swimming, carried along, etc.

Eyelash.-Half credit for "hair over eye" if child points to eyebrow instead of lashes.

The distributions of total scores on the vocabulary test, and of chronological and mental ages at the time of examination, are shown 
in Tables I, II and III. Table IV gives the data concerning each word of the vocabulary, including the number of times each was given and the number of successes, of failures, and of half credits in the kindergarten and the lower and upper Grade $I$. With the present word order, the following words, unknown to any of these children, were needlessly asked:

\begin{tabular}{|c|c|c|c|}
\hline $\begin{array}{l}\text { Frabt Coldum } \\
\text { 16. skill } \ldots \ldots \ldots \ldots\end{array}$ & $\begin{array}{c}\text { Trmes A8red } \\
35\end{array}$ & $\begin{array}{l}\text { Sacond Colum } \\
\text { 19. forfeit } \ldots \ldots \ldots\end{array}$ & $\begin{array}{c}\text { TrMeg ABxxd } \\
7\end{array}$ \\
\hline 17. ramble ..... & 18 & 20. sportive..... & 5 \\
\hline 18. civil ....... & 14 & 23. shrewd........ & 1 \\
\hline 21. juggler... ... & 7 & 24. repose...... & 1 \\
\hline 22. regard $\ldots \ldots \ldots$ & 4 & 25. peculiarity.... & 1 \\
\hline 23. stave........ & 1 & & \\
\hline 24. brunette...... & 1 & & \\
\hline 25. hysterics.... & 1 & & \\
\hline
\end{tabular}

On the basis of these figures, it is suggested that when the vocabulary test is given to young children the following word-order will be found useful, and will give greater certainty in making inferences as to when it is safe to omit the easiest words, and when the child has been carried far enough down the list. It also makes the two lists more nearly equal in difficulty, so that the use of one alone is somewhat more accurate. (It is my belief that this procedure is, however, almost never advisable.) Mimeographed sheets may easily be made up in this order, or in whatever similar order anyone wishes to derive from the data.

\begin{tabular}{|c|c|c|c|}
\hline & Caedrtb & & Cakbits \\
\hline 1. straw. $\ldots \ldots \ldots \ldots$ & 104 & 1. orange.. & 106 \\
\hline 2. envelope......... & 103 & 2. bonfire $\ldots \ldots \ldots \ldots$ & 97 \\
\hline 3. gown $\ldots \ldots \ldots \ldots$ & 83 & 3. puddle $\ldots \ldots, \ldots \ldots$ & 93 \\
\hline 4. tap........... & $871 / 2$ & 4. rule. $\ldots \ldots, \quad \ldots \quad \ldots$ & 81 \\
\hline 5. $\operatorname{scorch} \ldots \ldots \ldots$ & $731 / 2$ & 5. roar.... $\ldots \ldots \ldots$ & 73 \\
\hline 6. eyelash........... & 66 & 6. pork $\ldots \ldots \ldots$ & 55 \\
\hline 7. afloat.... ... . & 39 & 7. health.. . $\ldots . \ldots$ & 45 \\
\hline 8. impolite. .... ... & 32 & 8. plumbing $\ldots \ldots \ldots$ & 32 \\
\hline 9. copper........... & 23 & 9. haste... . . . . . & 23 \\
\hline 10. nerve............ & 14 & 10. guitar............ & 21 \\
\hline 11. dungeon $\ldots \ldots \ldots$ & $91 / 2$ & 11. muzzle. .. $\ldots$. & 13 \\
\hline 12. curse $\ldots \ldots \ldots \ldots \ldots$ & 9 & 12. $\operatorname{misuse} . \ldots \ldots \ldots$ & 7 \\
\hline 13. southern..... . . & 7 & 13. $\operatorname{snip} \ldots \ldots \ldots \ldots$ & 6 \\
\hline 14. lecture.. . . . . . . . & 4 & 14. reception $\ldots \ldots \ldots \ldots$ & $41 / 2$ \\
\hline 15. mellow . . . . . . & 4 & 15. noticeable . . . . & $31 / 2$ \\
\hline 16. insure $\quad \ldots \ldots$ & 3 & 16. quake..... . . . & $21 / 2$ \\
\hline 17. outward. ........ & $21 / 2$ & 17. treasury ........... & $21 / 2$ \\
\hline 18. apish $\ldots \ldots \ldots \ldots \ldots$ & 2 & 18. crunch $\ldots \ldots \ldots \ldots \ldots$ & 1 \\
\hline 19. ramble. $\ldots \ldots \ldots$ & $\mathbf{0}$ & 19. majesty $\ldots . . . \ldots \ldots$ & \\
\hline
\end{tabular}


Table I.-Dibtribdtion of Total Scoreg, Vocabduary

\begin{tabular}{|c|c|c|c|c|c|c|c|c|c|c|c|c|c|c|c|c|c|c|c|c|c|c|c|c|}
\hline Total scores & 4 & 5 & 6 & 7 & & 8 & 9 & 10 & 11 & 12 & & 13 & 14 & 15 & 16 & & 17 & 18 & 19 & 20 & 2 & & 22 & 23 \\
\hline 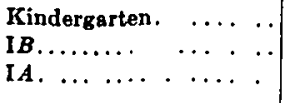 & $\begin{array}{l}1 \\
\cdot\end{array}$ & $\dot{j}$ & $\begin{array}{l}\mathbf{3} \\
\mathbf{3} \\
\cdots\end{array}$ & 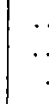 & & $\begin{array}{l}\mathbf{4} \\
\mathbf{3} \\
\mathbf{5}\end{array}$ & $\begin{array}{l}6 \\
3 \\
2\end{array}$ & $\begin{array}{l}2 \\
4\end{array}$ & $\begin{array}{l}\ddot{6} \\
7\end{array}$ & $\begin{array}{l}1 \\
5 \\
7\end{array}$ & & $\begin{array}{l}1 \\
4 \\
1\end{array}$ & $\begin{array}{l}1 \\
2 \\
5\end{array}$ & $\begin{array}{l}\dddot{3} \\
\mathbf{3}\end{array}$ & $\begin{array}{l}1 \\
1 \\
5\end{array}$ & & $\begin{array}{l}\dddot{2} \\
3\end{array}$ & $\ddot{3}$ & $\begin{array}{r}* \\
\ddot{*}\end{array}$ & $\begin{array}{l}1 \\
1 \\
1\end{array}$ & i & & $\ddot{1}$ & $\begin{array}{l}\ddot{1} \\
\ddot{\prime}\end{array}$ \\
\hline Total.. . . . & 1 & 1 & 6 & 0 & & 12 & 11 & 6 & 13 & 13 & & 6 & 8 & 6 & 7 & & 5 & 3 & 0 & 3 & 1 & & 3 & 1 \\
\hline \multicolumn{25}{|c|}{ Table II.-Distribution } \\
\hline Mental ages & & & $5^{8}$ & 510 & $6^{0}$ & $6^{2}$ & 64 & $6^{8}$ & $0^{8}$ & $6: 0$ & $7^{0}$ & $7^{2}$ & 74 & 74 & 78 & 710 & $8^{\circ}$ & $8:$ & 84 & 8• & $8^{8}$ & $8^{10}$ & 90 & 93 \\
\hline $\begin{array}{l}\text { Kindergarten } \\
\text { I } B\end{array}$ & 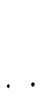 & . & $\begin{array}{l}2 \\
. \\
.\end{array}$ & 1 & $\begin{array}{l}4 \\
1\end{array}$ & $\begin{array}{l}2 \\
1\end{array}$ & $\begin{array}{l}3 \\
1 \\
1\end{array}$ & $\begin{array}{l}1 \\
2 \\
3\end{array}$ & $\begin{array}{l}4 \\
1 \\
3\end{array}$ & $\begin{array}{l}1 \\
3 \\
4\end{array}$ & $\begin{array}{l}1 \\
4 \\
5\end{array}$ & $\begin{array}{l}4 \\
4\end{array}$ & $\begin{array}{r}4 \\
10\end{array}$ & $\begin{array}{l}3 \\
8\end{array}$ & $\begin{array}{l}4 \\
2\end{array}$ & $\begin{array}{l}2 \\
0\end{array}$ & $\begin{array}{l}3 \\
4\end{array}$ & $\begin{array}{l}2 \\
1\end{array}$ & $\begin{array}{l}\mathbf{0} \\
\mathbf{3}\end{array}$ & $\begin{array}{l}2 \\
1\end{array}$ & 0 & 0 & $\mathbf{0}$ & 1 \\
\hline \multirow[t]{2}{*}{ Total .... . . } & & & 2 & 1 & 5 & 3 & 5 & 6 & 8 & 8 & 10 & 8 & 14 & 11 & 0 & 2 & 7 & 3 & $\mathbf{3}$ & 3 & 0 & 0 & 0 & 1 \\
\hline & $A B D$ & I & - & Ist & RIB & UTIO & OF & $\mathrm{Cr}$ & RON & OLOG & GICAI & IL A & GES & AT? & TIMe & OF & Ex & AMI & ratic & & & & & \\
\hline Chronological ages & $5^{2}$ & 54 & 68 & $5^{8}$ & $5^{10}$ & $6^{0}$ & $6^{2}$ & 04 & $6^{\circ}$ & $6^{\circ}$ & $6^{10}$ & $7^{0}$ & 72 & 74 & 74 & 78 & 710 & $8^{\circ}$ & 82 & $8^{4}$ & $8^{\circ}$ & $8^{8}$ & $8^{10}$ & 90 \\
\hline $\begin{array}{l}\text { Kindergarten } \ldots . \\
\text { IB } \ldots \ldots, \ldots, \\
\text { IA. } \ldots, \quad \cdots\end{array}$ & $\begin{array}{l}1 \\
. \\
\cdots\end{array}$ & $\begin{array}{l}3 \\
.\end{array}$ & $\begin{array}{l}1 \\
\cdots \\
\cdots\end{array}$ & 4 & $\begin{array}{l}4 \\
1 \\
.\end{array}$ & $\begin{array}{l}3 \\
5 \\
.\end{array}$ & $\begin{array}{l}2 \\
1 \\
.\end{array}$ & $\begin{array}{r}0 \\
3 \\
\cdots\end{array}$ & $\begin{array}{l}1 \\
9 \\
1\end{array}$ & $\begin{array}{l}1 \\
8\end{array}$ & $\begin{array}{r}9 \\
11\end{array}$ & $\begin{array}{l}2 \\
7\end{array}$ & $\begin{array}{l}2 \\
6\end{array}$ & $\begin{array}{l}2 \\
3\end{array}$ & $\begin{array}{l}0 \\
6\end{array}$ & $\begin{array}{l}1 \\
2\end{array}$ & $\begin{array}{l}0 \\
3\end{array}$ & $\begin{array}{l}0 \\
1\end{array}$ & $\begin{array}{l}0 \\
0\end{array}$ & $\begin{array}{l}0 \\
1\end{array}$ & $\begin{array}{l}1 \\
0\end{array}$ & 0 & 0 & 1 \\
\hline Total........... & 1 & 3 & 1 & 4 & 5 & 8 & 3 & 3 & 11 & 9 & 20 & 9 & 8 & 5 & 8 & 3 & $\mathbf{3}$ & 1 & $\mathbf{0}$ & 1 & 1 & 0 & 0 & 1 \\
\hline
\end{tabular}


TABLA IV

First Column

\begin{tabular}{|c|c|c|c|c|c|c|c|c|c|c|c|c|c|c|}
\hline \multirow{2}{*}{ Word order on blank } & \multicolumn{3}{|c|}{ Full oredit } & \multicolumn{3}{|c|}{ Half credit } & \multicolumn{3}{|c|}{ No credit } & \multicolumn{3}{|c|}{ Times asked } & \multirow{2}{*}{$\begin{array}{l}\text { Total } \\
\text { credits }\end{array}$} & \multirow{2}{*}{$\begin{array}{c}\text { Total times } \\
\text { asked }\end{array}$} \\
\hline & $\begin{array}{c}\text { Kinder- } \\
\text { garten }\end{array}$ & I $B$ & $I A$ & $\begin{array}{l}\text { Kinder- } \\
\text { garten }\end{array}$ & IB & IA & $\begin{array}{l}\text { Kinder- } \\
\text { garten }\end{array}$ & IB & I $A$ & $\begin{array}{l}\text { Kinder- } \\
\text { garten }\end{array}$ & I $B$ & I A & & \\
\hline 1. gown.. & 14 & 28 & 41 & . & $\cdots$ & . & 5 & 9 & 9 & 19 & 37 & 50 & 83 & 100 \\
\hline 2. $\operatorname{tap} \ldots$ & 12 & 29 & 46 & . & 1 & . & 7 & 7 & 4 & 19 & 37 & 50 & $871 / 2$ & 108 \\
\hline 3. scorch & 12 & 25 & 35 & 1 & . & 2 & 6 & 12 & 13 & 19 & 37 & 50 & $73 \%$ & 108 \\
\hline 4. puddle. & 18 & 32 & 43 & . & . & . & 1 & 5 & 7 & 19 & 37 & 50 & 03 & 100 \\
\hline 5. envelope & 18 & 36 & 49 & . & .. & $\cdots$ & 1 & 1 & 1 & 19 & 37 & 50 & 103 & 108 \\
\hline 6. rule.... & 13 & 26 & 42 & .. & . & .. & 6 & 11 & 8 & 18 & 37 & 50 & 81 & 106 \\
\hline 7. heslth... & 7 & 10 & 22 & . & . & .. & 12 & 21 & 28 & 18 & 37 & 50 & 45 & 108 \\
\hline 8. eye-lash.. . & 8 & 24 & 26 & 1 & 6 & 9 & 10 & 7 & 15 & 19 & 37 & 50 & $G B$ & 106 \\
\hline 9. copper... & 4 & 8 & 8 & 2 & 1 & 3 & 10 & 28 & 39 & 16 & 37 & 50 & 23 & 103 \\
\hline 10. curse...... & 1 & 4 & 4 & .. & & . & 10 & 33 & 46 & 11 & 37 & 50 & 9 & 98 \\
\hline 11. pork..... & 0 & 10 & 20 & 1 & 22 & 27 & 0 & $\mathbf{5}$ & 3 & 1 & 37 & 50 & 55 & 88 \\
\hline 12. outward.. & 0 & 1 & $\mathbf{0}$ & .. & $\mathbf{2}$ & 1 & . & 25 & 37 & . & 28 & 38 & $21 / 2$ & 66 \\
\hline 13. southern. . & .. & 0 & 5 & $\cdot$ & 2 & 2 & .. & 20 & 31 & . & 28 & 38 & 7 & 60 \\
\hline 14. leoture.... & .. & 1 & 3 & $\cdot$ & $\cdots$ & .. & . & 25 & 35 & . & 26 & 38 & 4 & 64 \\
\hline 15. dungeon. . & $\cdots$ & 3 & 6 & . & 1 & -. & .. & 22 & 33 & & 28 & 39 & $91 / 2$ & 65 \\
\hline 16. skill. .... & . & 0 & $\mathbf{0}$ & $\cdot$ & & .. & . & 16 & 19 & . & 16 & 19 & 0 & 85 \\
\hline 17. ramble.... & . & 0 & 0 & . & . & . & $\ldots$ & 7 & 11 & . & 7 & 11 & 0 & 18 \\
\hline 18. civil.... & . & 0 & 0 & - & . & . & . & 5 & 9 & . & 5 & 9 & $\mathbf{0}$ & 14 \\
\hline 19. insure.. & . & 1 & 1 & .. & 1 & 1 & . & 12 & 23 & . & 14 & 25 & $\mathbf{3}$ & 39 \\
\hline 20. nerve.. & .. & 0 & 3 & $\cdot$ & $\theta$ & 13 & .. & $\sigma$ & 11 & . & 14 & 27 & 14 & 41 \\
\hline 21. juggler... & . & 0 & $\mathbf{0}$ & . & .. & . & .. & 1 & 6 & .. & 1 & 6 & 0 & 7 \\
\hline 22. regard... & . & $\mathbf{0}$ & 0 & .. & .. & -. & 11 & 1 & 3 & .. & 1 & $\mathbf{3}$ & 0 & 4 \\
\hline
\end{tabular}


Becond Column

\begin{tabular}{|c|c|c|c|c|c|c|c|c|c|c|c|c|c|c|}
\hline \multirow{2}{*}{ Word order on blank } & \multicolumn{3}{|c|}{ Full credit } & \multicolumn{3}{|c|}{ Half crodit } & \multicolumn{3}{|c|}{ No credit } & \multicolumn{3}{|c|}{ Times asked } & \multirow{2}{*}{$\begin{array}{l}\text { Total } \\
\text { credits }\end{array}$} & \multirow{2}{*}{$\begin{array}{c}\text { Total times } \\
\text { asked }\end{array}$} \\
\hline & $\begin{array}{c}\text { Kinder- } \\
\text { garten }\end{array}$ & IB & IA & $\begin{array}{c}\text { Kinder- } \\
\text { garten }\end{array}$ & $I B$ & IA & $\begin{array}{c}\text { Kinder- } \\
\text { garten }\end{array}$ & I $B$ & IA & $\begin{array}{l}\text { Kinder- } \\
\text { garten }\end{array}$ & I $B$ & IA & & \\
\hline 1. orange.... .. & 19 & 37 & 50 & & .. & $\because$ & .. & - & .. & 19 & 37 & 50 & 106 & 108 \\
\hline 2. bonfire.. .. .. & 10 & 36 & 45 & $\theta$ & .. & $\mathbf{3}$ & .. & 1 & 2 & 19 & 37 & 50 & 97 & 106 \\
\hline 3. atraw...... & 18 & 37 & 49 & & .. & .. & 1 & & 1 & 19 & 37 & 50 & 104 & 108 \\
\hline 4. roar.. ... .. . & 8 & 27 & 37 & .. & .. & .. & 11 & 10 & 13 & 19 & 37 & 50 & 72 & 108 \\
\hline 5. haste... . . . . & 1 & 1 & 15 & 1 & 1 & 10 & 17 & 35 & 25 & 18 & 37 & 50 & 23 & 108 \\
\hline 6. afloat...... & 2 & 6 & 15 & 7 & 11 & 14 & 10 & 20 & 21 & 18 & $\mathbf{3 7}$ & 50 & 39 & 108 \\
\hline 7. guitar.. . . . . . & 4 & $\mathbf{5}$ & 12 & .. & . & .. & 15 & 32 & 38 & 10 & 37 & 50 & 21 & 106 \\
\hline 8. mellow $\ldots \ldots$. & 2 & 1 & 1 & .. & .. & & 14 & 36 & 48 & 16 & 37 & 49 & 4 & 102 \\
\hline 9. impolite. ....... & 3 & 13 & 15 & .. & 2 & .. & 11 & 21 & 34 & 14 & 30 & 49 & 32 & 89 \\
\hline 10. plumbing.. . ... & 4 & 12 & 12 & 1 & 1 & B & 8 & 22 & 32 & 13 & 35 & 50 & 32 & 98 \\
\hline 11. noticeable... .. . & $\mathbf{0}$ & 1 & 1 & .. & 1 & 2 & 1 & 22 & 36 & 1 & 24 & 39 & $31 / 2$ & 64 \\
\hline 12. muzzle. $\ldots, \ldots$ & $\mathbf{0}$ & 4 & $\boldsymbol{9}$ & .. & .. & .. & 1 & 22 & 36 & 1 & 26 & 45 & 13 & 72 \\
\hline 13. quake............. & .. & $\mathbf{0}$ & 0 & . & 4 & 1 & .. & 10 & 21 & .. & 14 & 22 & $21 / 2$ & 30 \\
\hline 14. roception... ....... & .. & 2 & 2 & .. & 0 & 1 & .. & 10 & 21 & .. & 12 & 24 & $43 / 2$ & 36 \\
\hline 15. majesty $\ldots . \quad \ldots$ & · & 0 & 0 & .. & 0 & 1 & . & $\mathbf{8}$ & 10 & .. & 9 & 20 & 34 & 29 \\
\hline 16. treasury... .... & .. & 0 & 1 & .. & 1 & 2 & .. & 7 & 16 & .. & 8 & 19 & $21 / 2$ & 27 \\
\hline 17. misuse.... & .. & 0 & 7 & .. & .. & 0 & .. & 10 & 13 & .. & 10 & 20 & 7 & 30 \\
\hline 18. crunch.$\ldots \ldots \ldots$ & .. & 0 & 1 & .. & .. & .. & .. & 6 & 13 & .. & 6 & 14 & 1 & 20 \\
\hline 19. forfeit $\ldots \ldots \ldots$ & .. & 0 & 0 & .. & .. & .. & .. & 1 & 6 & .. & 1 & 6 & 0 & 7 \\
\hline 20. sportive........... & .. & $\mathbf{0}$ & 0 & .. & .. & .. & .. & 1 & 4 & .. & 1 & 4 & $\mathbf{0}$ & 5 \\
\hline 21 apish... $\ldots \ldots \ldots \ldots$ & .. & 2 & $\mathbf{0}$ & .. & .. & .. & .. & 1 & 11 & .. & 3 & 11 & 2 & 14 \\
\hline 22. snip $\ldots \ldots \ldots \ldots$ & .. & 4 & 2 & .. & .. & .. & .. & 3 & 15 & .. & 7 & 17 & 6 & 24 \\
\hline
\end{tabular}


The larger changes in order seem to be due to the fact that certain more abstract terms, such as afloat, haste, mellow, noticeable, outward and skill, are relatively more difficult at these early ages; and that some of the more concrete terms, such as dungeon, envelope, eyelash, nerve, plumbing, pork and snip, are learned earlier and are (relative to an older group having an equally small vocabulary) more likely to be known. Smaller changes may be due to the fact that the number of cases tabulated is small, though in general the lists from the subgroups confirm one another. The first four words appear in the same order with the kindergarten and $I B$ grouped together as with the I $A$ children; the first 13 words for the two groups are the same words, though the order is different. However, many tabulations are required before the best order for any limited group can be attained. It is suggested that others who have accumulated Stanford-Binet records for children of this age might tabulate and publish their data in such form that eventually they could be combined, and the best order finally determined. 